\title{
Using NSGAIl to Solve Bi-objective Bed Allocation Problems
}

\author{
Jalal A. Sultan ${ }^{1}$, Ban A. Mitras ${ }^{2}$,Raghad M. Jasim ${ }^{3}$ \\ ${ }^{1}$ Department of Mathematics, College of Al-Hamdaniah Education, University of Mosul, Al-Hamdaniah, Iraq \\ ${ }^{2}$ Department of Statistics \& Information, College of Mathematics and Computer Sciences, University of Mosul, Mosul, \\ Iraq \\ ${ }^{3}$ Department of Operation Research, College of Mathematics and Computer Sciences, University of Mosul, Mosul, Iraq \\ Jalalstat2011@gmail.com
}

\begin{abstract}
The Bed Allocation Problem (BAP) is NPcomplete and always high dimensional. In this paper, a biobjective decision aiding model based on queuing theory is introduced for allocation of beds in a hospital. The problem is modeled as an M/PH/n queue. The objectives include maximizing the patient admission rate human resources, in particular, maximization of the nursing work hours. The proposed model is solved by using Non-dominated Sorting Genetic Algorithm-II (NSGA-II), which is a very effective algorithm for solving multi-objective optimization problems and finding optimal Pareto front. The paper describes an application of the model, dealing with a public hospital in Iraq. The results related that multi-objective model was presented suitable framework for bed allocation and optimum use.
\end{abstract}

Keywords-Bed Allocation Problem; Non-Dominated Sorting; Genetic Algorithm; Multi-Objective Optimization.

\section{INTRODUCTION}

Busy hospital systems constantly provide new challenges to their managers and decision-makers due to high demands for service, high costs, limited budget and healthcare resources. As a result, decision-makers are continuously studying efficacy and efficiency of existing hospital systems and must be able to evaluate the outcomes of any changes they make to these systems [6]. Hospitals today are faced with several pressures such as increasing equipment costs, a shortage of qualified healthcare professionals and limited hospital facilities [7]. Bed shortage or improper bed distribution can be increase waiting times, patient displacement and distrust of bed management [8]. Hospital bed capacity planning accuracy is important to satisfy patients' needs, organize departments and improve the quality and amount of service provided.

In general, bed allocation problem concerns the permanent number of beds assigned to the different medical and surgical specialties in a hospital. An appropriate bed allocation is important for cost-efficiency of hospitals. Too few beds assigned to a specialty may lead to customer assignments to units with inappropriate equipment and inadequate staff training. This, in turn, would likely result in lower quality of care. Further, too many beds increases costs through an underutilization of resources [5].
Several studies in the literature focus on the problem of bed allocation across different departments, medical specialties, or types of patients in a hospital. Esogbue and Singh [1], for example, develop a mathematical model useful in devising an admission policy in a ward for different types of patients and in which the objective function is made up of shortage and holding costs. Kao and Tung [5] present an approach for periodically reallocating beds to services to minimize the expected overflows. Their demand forecasting system uses an $M / G / \infty$ queuing model to approximate the patient population dynamics for each service. Their study emphasizes that forecasts should form the basis for analysis and accepts approximation by queuing models for the sake of procedural simplicity. Lapierre et al. [18] consider the problem of allocating a number of beds to different medical and surgical specialties in a hospital, taking into account that the scheduling of medical procedures varies over a week and that the demand for various medical services shows seasonality. A time series model is developed by using hourly census data to make good decisions regarding the size of each unit. Similarly, to determine the frequency distribution associated with a hospital care units census, Cote [16] present an analytic approach based on a modified version of the HoltWinters multiplicative seasonality forecasting model.

Very few studies, however, consider the bed allocation problem as a multi-objective problem. Kim et al. [17] demonstrate with a validated computersimulation model that: there is no single dominant solution to the bed-allocation problem, thus indicating the multi-objective nature of the problem. Oddoye et al. [12] detail a GP model to analyze the performance of a Medical Assessment Unit (MAU) and find solutions for bed allocation for patients with minimum delay. However, due to the deterministic nature of the model built, it is difficult to investigate the systems performance for optimal work flow. Hence, Oddoye et al. [11] present a simulation model which consider the factors in length of stay, number of beds, nurses and doctors in the MAU. Thereafter a GP model is applied to perform trade-off analysis, using the results from the simulation model. The hourly allocation schemes of resources can be deployed 
within the MAU to help minimizing delays and increase the flow of patients.

In recent years, the bed allocation problem (BAP) has received increasing attentions. Kinds of mathematical models for BAP were built in the literature, among which the uses of queuing theory are very common. In [7] and [8], the model of $M / P H / n$ queues was proposed, where $M$ denotes patient arrival subject to Poisson distribution (Markov arrivals), $P H$ denotes patient lengths of stay $(L o S)$ subject to Phase-type distribution, and $n$ is the number of beds.

This paper introduces a bi-objective decision aiding model based on queuing theory for allocation of beds in a general hospital. It is assumed that the number of beds affects the performance of a department in terms of (1) the acceptance probability when a new patient arrives and (2) the nursing work hours.

The paper is organized as follows: Section 2 present the proposed model of bed allocation problem. The multiobjective genetic algorithm NSGAII to solve BAP has been proffered in Section 3. The results and discussion are given on Section 4. The conclusion of this study has been presented in Section 5.

\section{THE PROPSED MODEL OF BAP}

In this study, important objectives and constraints of beds allocation are identified through the review of literature and interview with experts. The model contains two objective functions and three constraints. The sets of objectives and constraints are given below.

According to Gorunescu et al. [7], the model of BAP is defined as a $M / P H / n$ queue. Suppose that the hospital has $n$ beds and $D$ departments. For each department $i, i=1,2, \ldots$ ,D, the number of beds is $n_{i}$, the Poisson arrival rate is $\lambda_{i}$, and the mean of length of stay which is calculated by the Phase-type distribution is $\mu_{\mathrm{i}}$, We deduce that the mean number of arrivals during a length of stay is $\lambda_{i} \mu_{i}$. Thus, according to Erlang's loss formula, the probability $P_{\mathbb{i}}$ that some arrivals are lost because all $n_{i}$ beds are occupied can be given by Equation (1).

$P_{i}=\left[\left(\lambda_{i} \mu_{i}\right)^{n_{i}} / n_{i} l\right] / \sum_{j=0}^{n_{i}}\left[\left(\lambda_{i} \mu_{i}\right)^{j} / j !\right]$

Where $j$ is defined as the number of phases of the service time. Therefore, we deduce the admission rate of patient in department $i$ as

$P a_{\mathrm{i}}=1-P_{\mathrm{i}}$

The total nursing hours in department $i$, can be represented by Equation (3):

$F n_{i}=t s_{i} \times n_{i}$

Where $t s_{i}$ is nursing hours for each bed in department $i$. The model constraint set (4)-(6) is shown below. The constraint (4) make sure that the total number of beds $(N)$ is equal to the sum of beds in each department. The second constraint makes sure that the total nursing hours between $\mathrm{H}$ and $\mathrm{L}$, where $\mathrm{H}$ is maximum hour of nursing with most overtime and $\mathrm{L}$ is minimum hour of nursing with the Least overtime. The last constraint makes sure that there is no department without beds.
$\sum_{i=0}^{D} n_{i}=N$

$L \leq \sum_{i=0}^{D} F n_{i} \leq H$

$n_{i}>0, i=1,2, \ldots, D$

\section{Multiobjective Genetic AlgORITM For BAP}

Multi-objective optimization is a research topic attracting much attention because many optimization problems involve multiple and conflicting objectives and a compromise may have to be made among these objectives. The most important concept in multiobjective optimization is Pareto optimality. A solution is Pareto optimal if it is not dominated by any other solution in terms of all objectives considered [2]. Since a number of solutions may be Pareto optimal, the task of multiobjective optimization is to find as many as possible such non dominated solutions, and this task is quite complex. In this section, a simulation-based multi-objective optimization method that combines simulation evaluation of performance and meta-heuristic search with multiobjective genetic algorithms called NSGA-II is adapted for the optimization of bed allocation. We use a multiobjective optimization method because there are more than one objective in bed allocation optimization especially patient admission rate and human resources.

GA is a search technique based on the mechanism of natural selection and reproduction introduced by Holland and is used to search large, non-linear solution spaces where expert knowledge is lacking or difficult to encode and where traditional optimization techniques fall short. It starts with an initial set of randomly generated solutions called population and each individual in the population is called chromosome that is representing a solution. Each chromosome is composed of a set of elements called genes. At each iteration (generation) of GA, all newly generated chromosomes are evaluated using a fitness function to determine their qualities. High quality chromosomes are selected to produce offspring chromosomes through genetic operators, namely, crossover and mutation. After a number of generations, the GA converges to a chromosome which is very likely to be an optimal solution or a solution close to the optimum [3].

\subsection{NSGA II Principle}

NSGA-II computes successive generations of a population of solutions belonging to non-dominated fronts. The non-dominated set is identified and constitutes the non-dominated front of level 1 or front 1 . In order to find the individuals in the next non-dominated front, the solutions of front 1 are discounted temporarily and the above procedure is repeated. This process continues until all fronts are identified. In order to maintain diversity in the population, the crowdingdistance is used. The overall structure of the NSGAII is specified by Algorithm 1 [13].

Algorithm 1: NSGAII overall structure

Create the initial population $P$ of size $n$

Evaluate the $n$ solutions using simulation

Sort $P$ by non domination 
Compute the crowding distance of each solution while Stopping Criterion do

Create and add $n$ children to $P$ (using genetic operators: selection, crossover and mutation of two parents)

Sort $P$ by non domination

Compute the crowding distance of each solution

newP $=\emptyset$

$i=1$

while $\mid$ new $P|+|$ front (i) $\mid \leq \mathrm{n}$ do

Add front $(i)$ to newP

$i=i+1$

end while

missing $=n-\mid$ new $P$

if missing $\neq 0$ then

Sort the solutions by descending order of the crowding distance

for $j=1$ to missing do

Add the $j$ th solution of front $(i)$ to newP

end for

$P=$ now $P$

end if

end while

\subsection{GA Components for Bi-objective Bed Allocation}

In this study, the BAP has two objectives, namely patient admission rate and nursing hours, to optimize. Basic operations that characterize our adapted NSGA-II are explained as follows.

\section{A. Encoding}

For BAP, only one parameter is involved which represent the number of beds $n_{i}$ in each department $i$. For a BAP with $D$ departments, each chromosome is encoded as

$$
\left[n 1, \ldots, n_{i}, \ldots, n_{D}\right] \text {. }
$$

\section{B. Initial Population}

The NSGA-II starts the search by generating a population of candidate solutions. In our implementation, this population is randomly generated according to the uniform distributions. First of all, we generate a random value $(R i)$ for each department $i$ from uniform distribution $U[0,1]$. Then calculating the number of beds in each department by using equations (7) and (8) respectively.

$n_{\mathrm{i}}=\operatorname{round}\left(\left[R_{i} / \sum_{j=1}^{D} R_{j}\right] \times N\right), i=1,2, \ldots, D-1$

$n_{i}=N-\sum_{j=1}^{D-1} n_{j}, i=D$

\section{Chromosomes Evaluation}

The admission rate of patient and the nursing hours are optimized simultaneously. The objective functions are defined as

$F_{1}=\sum_{i=1}^{D} w_{i} \times P a_{i}$
$F_{2}=\sum_{i=1}^{D} w_{i} \times F n_{i}$

Where $P a_{\mathrm{i}}$ and $F n_{\mathrm{i}}$ are the admission rate and bed occupancy in department $i$ according to (2) and (3); $w_{i}$ is the proportion of patients in department $i$ according to the statistical data in the hospital. In our study, each chromosome is evaluated through simulation. The simulation is controlled by two control parameters: the number of chromosomes and the number of iterations.

\section{Selection}

Selection is a process in which chromosomes are chosen according to their fitness function value or their rank value. In this study, the tournament parent selection is used. Tournament selection is one of selection methods in genetic algorithms which runs a tournament among a few individuals chosen randomly from the population and selects the winner (the one with the best fitness) for crossover. The tournament size used in our computational experiments is 2 .

\section{E. Crossover and Mutation}

The crossover produces new offspring chromosomes from parent individuals. Two new chromosomes are created by exchanging some genes of two parent chromosomes. We use in our implementation the singlepoint crossover. This kind of

crossover creates a pair of offspring by exchanging parts of the parents after a randomly chosen crossover point.

The mutation includes adding and subtracting one bed to different random genes in the chromosome. The function of mutation is to maintain the diversity of the population in order to prevent too fast convergence of the algorithm.

\section{F. Crowding Distance Calculation}

As the overall population size of $P$ is $2 n$, we cannot accommodate all fronts in the new parent population (newP) of size $n$. The non accommodated fronts are simply deleted. When the last allowed front is considered, there may exist more solutions in the last front than the remaining slots in the new population. In order to avoid arbitrarily choosing individuals, we choose the individuals that can assure diversity between the considered ones; that is what we call a niching strategy. For this reason, we calculate the crowding distance measuring the Euclidean distance between the neighboring individuals in each front (Fig. 1). The Algorithm 2 describes the crowding distance procedure [13].

\section{Algorithm 2: Crowding distance calculation}

Let $\bar{\zeta}$ the number of solutions

for each solution $i \overrightarrow{\text { do }}$

Initialize the distance to 0

for each objective $m$ do

Sort the solutions according to the objective $m$ 
$d_{1}=0, d_{\zeta}=\infty$

for $i=2$ to $(\zeta-1)$ do

$d_{i}=d_{i}+\left(f_{m}^{i+1}-f_{m}^{i-1}\right) /\left(f_{m}^{M A X}-f_{m}^{M I N}\right)$

end for

end for

end for

Where $f_{m}^{i}$ is the value of the objective function $m$ of the $i$ th solution. Thereafter, those strings with largest crowding distance values are chosen to become the new parent population. Once the non-dominated sorting is over, the new parent population, new $P$, is created by choosing solutions of different non-dominated fronts.

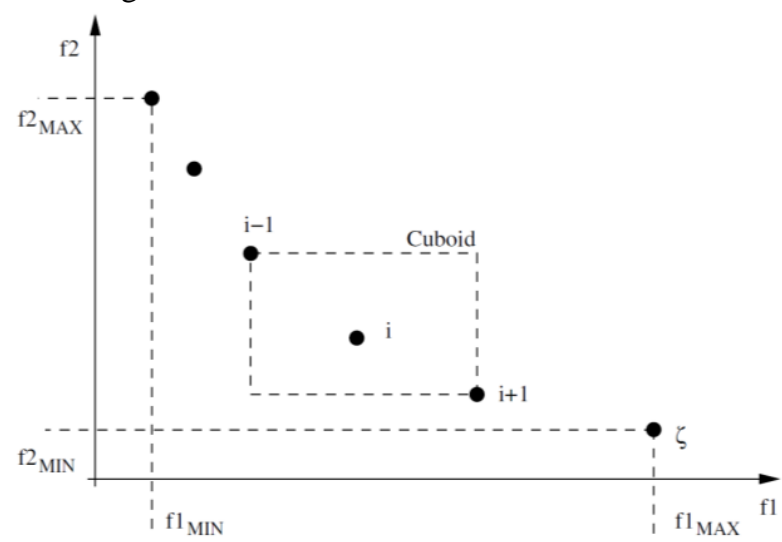

Fig. 1. Crowding distance

\section{G. Stopping Conditions}

There are no universal stopping conditions accepted for multi-objective genetic algorithms. In this study, we simply stop our algorithm after a given number of iterations $(\mathrm{Ng})$.

\section{RESUlTS AND DISSCUSIONS}

In this section, a real life bed allocation problem is presented. For confidential reason, the name of the hospital concerned is not mentioned. The hospital has 7 departments and 202 beds. The maximum and minimum hour of nursing with overtime are 200 hour and 150 hour respectively. The Poisson arrival rate, the mean of length of stay and nursing hours for each bed are shown in Table I. The parameters for NSGA-II after many experiments were a crossover probability $\left(p_{c}\right)$ equal to $0.8 ;$ a mutation probability $\left(p_{m}\right)$ equal to 0.3 ; the number of iterations $N_{g}=500$; the number of population is 80 .

TABLE I. ARRIVAL RATE, THE MEAN OF LOS AND NURSING HOURS FOR EACH BED

\begin{tabular}{|c|c|c|c|c|}
\hline$I$ & Department & $\lambda_{i}$ & $\mu_{i}$ & $\begin{array}{c}t s_{i} \\
(h)\end{array}$ \\
\hline 1 & General Surgery 1 & 18.115 & 10.3462 & 1.2 \\
\hline 2 & General Surgery 2 & 18.192 & 10.3846 & 1.15 \\
\hline 3 & General Surgery 3 & 18.538 & 12.156 & 1.25 \\
\hline 4 & Urology Surgery & 19.153 & 9.8077 & 0.65 \\
\hline 5 & Fascia Surgery & 4.461 & 2.1923 & 0.9 \\
\hline 6 & Orthopedics & 7.961 & 3.5385 & 0.55 \\
\hline 7 & ENT & 18 & 11.1538 & 0.6 \\
\hline
\end{tabular}

As the population size is set as 80,80 solutions are generated by the NSGAII for the hospital manager to select. Due to limitations of the paper length, the results are presented here only for the one-boundary solutions. We compare our solution with the previous allocation decision of their hospital. As shown in Table II, $n$ is the number of beds allocated for each department, $\mathrm{Pa}$ is the admission rate, and $F n$ is the nursing hours. Moreover, Fig. 2 shows the changes in performance of the patient admission rate in each department, while Fig. 3 shows the changes in performance of the nursing hours in each department. The advantages of the allocation decision generated by NSGAII are summarized as follows.

TABLE II. COMPARISON BETWEEN THE NEW AND PREVIOUS ALLOCATION DECISIONS

\begin{tabular}{|c|c|c|c|c|c|c|}
\hline \multirow{2}{*}{ Dept. } & \multicolumn{3}{|c|}{ Previous } & \multicolumn{3}{|c|}{ New } \\
\cline { 2 - 7 } & $\boldsymbol{N}$ & $\boldsymbol{P a}$ & $\boldsymbol{F n}$ & $\boldsymbol{n}$ & $\boldsymbol{P a}$ & $\boldsymbol{F n}$ \\
\hline 1 & 32 & $66.34 \%$ & 38.40 & 40 & $\begin{array}{c}80.6 \\
8 \%\end{array}$ & 48.00 \\
\hline 2 & 32 & $64.36 \%$ & 36.80 & 40 & $\begin{array}{c}78.5 \\
7 \%\end{array}$ & 46.00 \\
\hline 3 & 32 & $54.80 \%$ & 40.00 & 34 & $\begin{array}{c}58.0 \\
8 \%\end{array}$ & 42.50 \\
\hline 4 & 32 & $64.68 \%$ & 20.80 & 34 & $\begin{array}{c}68.4 \\
0 \%\end{array}$ & 22.10 \\
\hline 5 & 10 & $99.98 \%$ & 9.00 & 7 & $\begin{array}{c}99.1 \\
0 \%\end{array}$ & 6.30 \\
\hline 6 & 32 & $100 \%$ & 17.60 & 13 & $\begin{array}{c}100 \\
\%\end{array}$ & 7.15 \\
\hline 7 & 32 & $60.94 \%$ & 19.20 & 34 & $\begin{array}{c}64.5 \\
1 \%\end{array}$ & 20.40 \\
\hline Total & 202 & $73.01 \%$ & 181.80 & 202 & $\begin{array}{c}78.4 \\
8 \%\end{array}$ & 192.45 \\
\hline
\end{tabular}

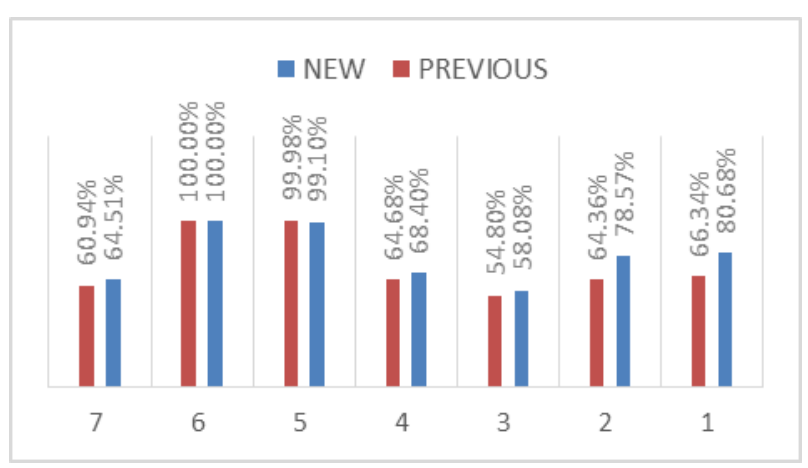

Fig. 2. Comparisons of patient admission rates

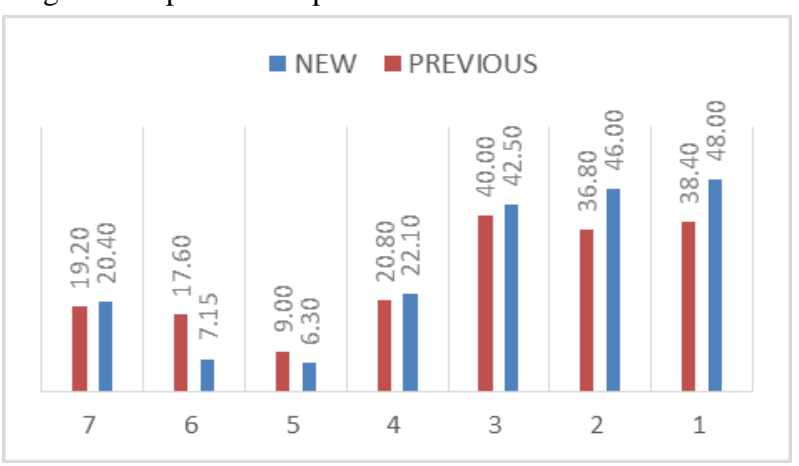

Fig. 3. Comparisons of nursing hours 
The NSGAII balances the resource competition among different departments. The working efficiency and level of management in the hospital are improved. In the previous allocation decision, department 6 has the highest admission rate about $100 \%$ and department 3 has the lowest admission rate about $54.80 \%$. In contrast, in the new allocation the highest admission rate is $100 \%$ in department 6 and the lowest admission rate is $58.08 \%$ in department 7 . Therefore, in the new allocation, decisions, not only the admission rates are balanced, but also the lowest admission rates are remarkably improved. Almost, similar results are also reported in nursing hours. As can be observed, the highest nursing hours is 40 hour in the previous situation, 48 in the new situation.

From an overall point of view, the general patient admission rate and nursing hours are increased from $73.01 \%$ and 181.8 to $78.48 \%$ and 192.45 respectively.

\section{CONCLUSIONS}

This paper proposes a bi-objective decision aiding model based on queuing theory for BAP in a hospital. The Non-dominated Sorting Genetic Algorithm-II (NSGAII) is used to optimize the proposed model. Solved by NSGAII, the beds in 202-general hospital in Iraq, is reallocated. Simulation results show that resource competition among different departments is more balanced and the general patient admission rate and nursing hours in the hospital both are increased. Thus, the service level and the resource usage in the hospital are improved simultaneously.

\section{References}

[1] A. Esogbue, A. Singh "A stochastic model for an optimal priority bed distribution problem in a hospital ward. Oper Res, vol. 24. No. 5, pp884-898,1976.

[2] A. Coello Coello, B. Lamon, A. Van Veldhuisen "Evolutionary Algorithms for Solving Multi-Objective Problems", Springer, 2007.

[3] B. Tomoiagă, M. Chindriş, A. Sumper, A. Sudria-Andreu, R. Villafafila-Robles, "Pareto Optimal Reconfiguration of Power Distribution Systems Using a Genetic Algorithm Based on NSGA-II. Energies" vol. 6, pp. 1439-1455, 2003.

[4] D. J. Worthington and A. Wall, "Using the Discrete Time Modelling Approach to Evaluate the Time-Dependent Behaviour of Queueing Systems," The Journal of the Operational Research Society, vol. 50, no. 8, pp. 777-788, 1999.

[5] E. P. C. Kao and G. G. Tung, "Bed Allocation in a Public Health Care Delivery System," Management Science, vol. 27, no. 5, pp. 507-520, 198U.

[6] E. P. C. Kao and G. G. Tung, "Bed Allocation in a Public Health Care Delivery System", Management Science, vol. 27, no. 5, pp. 507-520,1981.

[7] F. Gorunescu, S. I. McClean and P. H. Millard, "A Queueing Model for Bed-Occupancy Management and Planning of Hospitals," The Journal of the Operational Research SOCiety, vol. 53, no. I, pp. 19-24, 2002.

[8] F. Gorunescu, S. I. McClean and P. H. Millard, "Using a Queueing Model to Help Plan Bed Allocation in a Department of Geriatric Medicine," Health Care Management Science, vol. 5, no. 4, 2002.

[9] H. R. Feili, "Improving the Health Care Systems Performance by Simulation Optimization,” J. Math. Comput. Sci., vol. 7, pp. 7379, 2013.
[10] J. C Ridge, S. K. Jones, M. S. Nielsen and A. K. Shahani, "Capacity planning for intensive care units," European Journal of Operational Research, vol. 105, no. 2, pp. 346-355, 1998.

[11] J. Nguyen, P. Six, D. Antonioli, P. Glemain, G. Potel, P. Lombrail and P. Le Beux, "A simple method to optimize hospital beds capacity," International Journal of Medical 11iformatics, vol. 74, no. I, pp. 39-49, 2005.

[12] J. Oddoye, M. Tamiz, D. Jones, P. Schmidt "A simulation model for health planning in a medical assessment unit with multiobjective output analysis". Technical report, Management Mathematics Group, University of Portsmouth, UK, 2007.

[13] J. Oddoye, M. Yaghoobi, M. Tamiz, D. Jones, P. Schmidt "A multi-objective model determine efficient resource levels in a medical assessment unit". J Oper Res Soc, 2007.

[14] K. Deb, A. Agarwal, T. Meyarivan, "A fast and elitist multiobjective genetic algorithm: NSGA-II". IEEE Transactions on Evolutionary Computation vol. 6, no. 2, pp. 182-202, 2002.

[15] L. V. Green and V. Nguyen, "Strategies for cutting hospital beds: the impact on patient service," Health Services Research, vol. 36, no. 2, pp. 421-442,2001.

[16] L. Green, "Queueing Analysis in Healthcare", Patient Flow: Reducing Delay in Healthcare Delivery, pp. 281-308, 2006.

[17] M. Cote, "A note on bed allocation techniques based on census data". Socioecon Plan Sci, vol. 39, pp183-192, 2005.

[18] S. C. Kim, I. Horowitz, K. K. Young and T. A. Buckley, "Flexible bed allocation and performance in the intensive care unit," Journal of Operations Management, vol. 18, no. 4, pp. 427-443, 2000

[19] S. Lapierre, D. Goldsman, R. Cochran, J. DuBow "Bed allocation techniques based on census data", Socioecon Plan Sci, vol. 33pp. 25-38, 1999.

[20] S. M. Ballard and M. E. Kuhl, "The use of simulation to determine maximum capacity in the surgical suite operating room," in Proceedings - Winter Simulation Conference, vol. 7, pp. $433-$ 438,2006.

[21] T. Ibarraki and N. Katoh, Resource Allocation Problems, The MIT Press, Cambridge, MA, 1988.

[22] X.-D. Li, P. Beullens, D. Jones and M. Tamiz, "Optimal Bed Allocation in Hospitals," Lecture Notes in Economics and Mathematical Systems, vol. 618, pp. 253-265, 2009.

[23] Y. Tütüncü and D. Newlands, "Hospital Bed Capacity and Mix Problem for State Supported Public and Fee Paying Private Wards," IÉSEG Sch. Manag. CNRSLEM (UMR 8179), no. Umr 8179, 2009. 